\title{
Fuzzy Sliding Mode Control for Inverted Pendulum with Random Disturbance
}

\author{
Jieke Lin, Guanglin Shi, Shu Tang \\ School of Mechanical Engineering/ Shanghai Jiao Tong University \\ 800 Dongchuan RD. Minhang District, Shanghai, China \\ 52281injieke@sjtu.edu.cn; glshi@263.net; tsoup1995@sjtu.edu.cn
}

\begin{abstract}
In this paper, a novel inverted pendulum with a motor-driven random disturbance rod at the top is presented, which is different from the normal inverted pendulum. The simplified mathematic model of the control system is established on the basis of dynamic equations. The traditional PID control method is adopted and proved invalid for this system. Then Sliding Mode Control is used for the system due to its strong anti-interference ability. To weakening the chattering which is common in Sliding Mode Control, Fuzzy Sliding Mode Control is developed using fuzzy inference to adjust the thickness of the boundary layer in real time. A simulation of the control system is produced by combing the software MATLAB/Simulink and Adams, which can real-time display the movement of the system during simulation. The theoretical analysis and simulation results verify the rationality of the proposed control method for the inverted pendulum with random disturbance.
\end{abstract}

Keywords: Inverted Pendulum, Random Disturbance, PID Control, Sliding Mode Control (SMC), Fuzzy Sliding Mode Control (Fuzzy SMC), MATALB/Simulink, Adams

\section{Introduction}

The inverted pendulum is a high-order, complex, unstable, strongly coupled nonlinear system that can be used to verify whether a control method has the ability to handle multivariable, nonlinear and absolutely unstable system so the inverted pendulum can be used as a typical control model to evaluate the control method [1].The inverted pendulum is also the basis for many other control design techniques such as the strategy used for generating gait pattern to control the robot walk like the human [2], the theory for mobile wheeled inverted pendulum control system design [3], the control method of underactuated robotic systems including aircraft, spacecraft, helicopters, underwater vehicles [4],etc. There are some types of inverted pendulum: one-stage inverted pendulum, double inverted pendulum, triple inverted pendulum, etc. Although uncertain external disturbances such as damping and friction can bring errors to the dynamic equation, the physical structures of these inverted pendulum systems do not change randomly ensuring the equilibrium position of the system is approximately constant [5 6]. Many control methods have been implemented on the inverted pendulum system such as: PID control [7], Nonlinear Control [8], Fuzzy Control [9], Robust Control [10], Neural Networks Control [11], etc.

Sliding Mode Control Algorithm has strong robustness which can continuously adjust the structure of the control system, and it is insensitive to the changes in dynamic equation parameters or external disturbances that may occur in the system. As a result, Sling Mode Control is often used for servo system control. The most significant problem in Sliding Mode Control is the chattering phenomenon near the equilibrium position [12], which is harmful to the mechanical structure but can be well weakened by combining the Sliding Mode Control with the Fuzzy Control. In this strategy, Fuzzy Inference method is used to adjust the thickness of the boundary layer, so as to eliminate the chattering phenomenon while maintaining the robustness of the sliding mode control [13 14]. The Fuzzy Sliding Mode Controller is proved able to ensure the hydraulic turbine regulating system work smoothly [15], lower the noise of the reluctance motor [16], reduce the speed tracking error of the hydraulic pressure coupling drive [17], which show good robust to nonlinear and strong inference systems.

In this paper, a new type of inverted pendulum with a motor-driven random disturbance rod at the top is presented. The physical structure of the pendulum can change randomly in real time by the random movements of the disturbance rod. Because of the changeable structure, the equilibrium position of the system is a changeable and uncertain value different from the normal inverted pendulum. Three control algorithms (PID Controller, Sliding Mode Controller, Fuzzy Sliding Mode Controller) are designed and used for the motion simulation by combining the software MATLAB/Simulink and Adams. 
The simulation results show that the Fuzzy Sliding Mode control can achieve a better performance in the inverted pendulum control system with random disturbance.

\section{Mechanism Design and Mathematical Model \\ 2.1. Mechanism Design}

The sketch of new inverted pendulum with a motor-driven random disturbance rod at the top is shown in Figure 1.This system mainly consists of two parts.

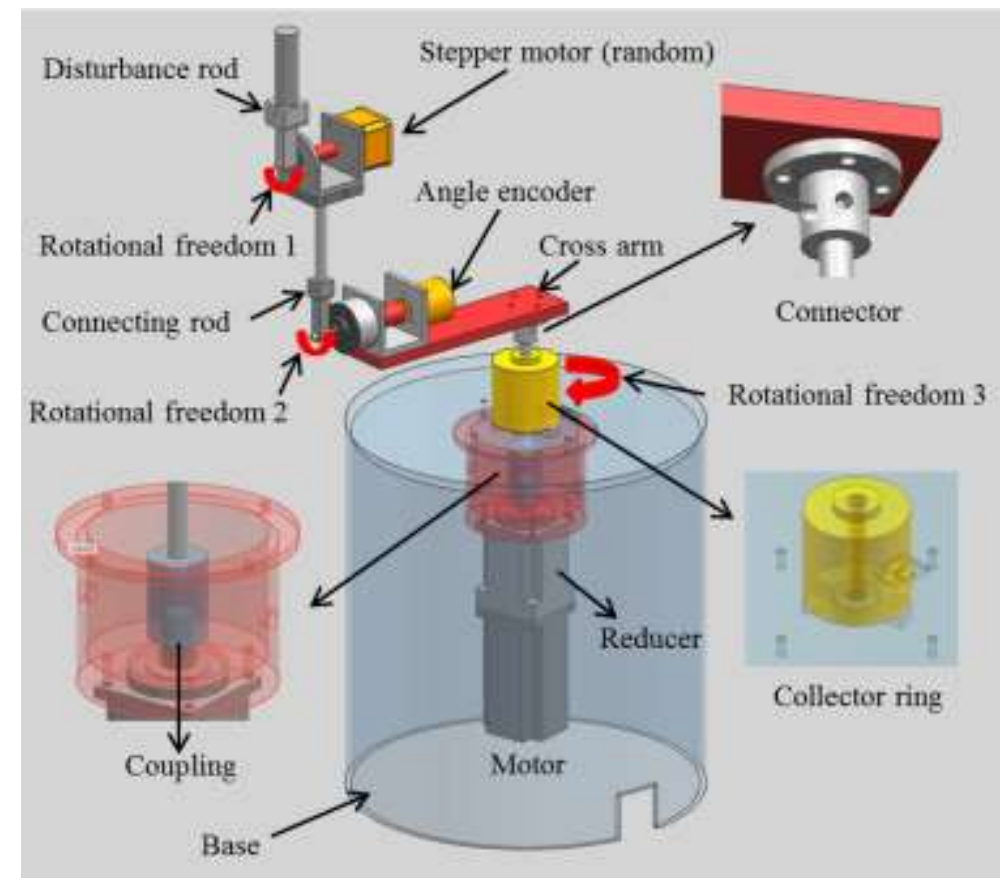

Fig. 1: Sketch of the Inverted Pendulum with Random Disturbance.

(1) Rotary control mechanism: It is mainly composed of AC servo motor, reducer, diversion ring and angle Encoder. The servo motor controls the rotation of the cross arm after decelerating through the reducer. The servo motor is driven by the servo motor driver which receives control information from the controller. The angle encoder can transmit the angle value of the connecting rod to the controller in real time.

(2) Random disturbance mechanism: It is mainly composed of a stepper motor and a motor-driven disturbance rod. The stepper motor drives the rod to randomly rotate which causes the physical structure of the pendulum changing in real time.

The control goal is to keep the angle between the connecting rod and the vertical direction within in a certain angle when there is a limited random disturbance caused by the disturbance rod.

\subsection{Mathematical Model}

The precise dynamic model equation of the inverted pendulum is very complicated and unpredictable because of the randomly unmeasurable disturbance. But the dynamic model equation is needed when designing the Sliding Mode Controller or Fuzzy Sliding Mode Controller. To meet the demand, a simplified model is proposed in Figure 2, ignoring the friction, damping. And the angle of the disturbance rod is set as $0^{\circ}$, assuming there is no random disturbance. 


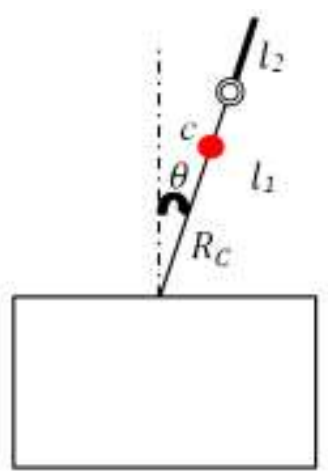

(a) Front View

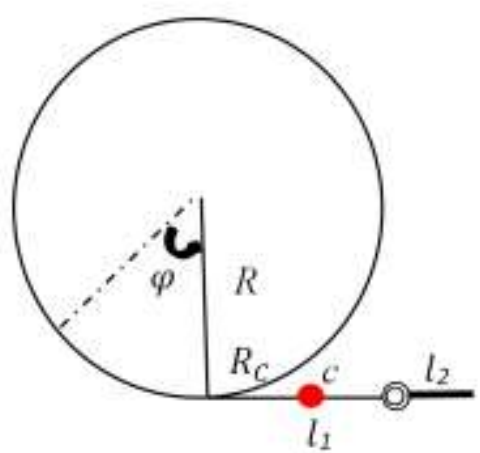

(b) Top View

Fig. 2: Simplified Inverted Pendulum Model with Random Disturbance.

As for the vertical swinging part of the inverted pendulum, all masses can be equivalent at centroid point(c) ignoring the quality of the connecting rod and the disturbing rod. By dynamic analysis, the dynamic equation is shown in Eq. (1).

$$
\ddot{\theta}=\frac{m\left(l_{1}, l_{2}\right) \cdot g \cdot R_{C}}{J_{C}\left(m, l_{1}, l_{2}\right)} \cdot \theta-\frac{m\left(l_{1}, l_{2}\right) \cdot R_{C} \cdot R}{J_{C}\left(m, l_{1}, l_{2}\right)} \ddot{\varphi}
$$

Where $m$ is the equivalent mass of the swinging part, $l_{1}$ is the distance from the connection point of the swinging part to the end point of the cross arm, $l_{2}$ is the length of the random disturbance rod, $R_{C}$ is the distance of from the centroid of the swinging part to the end point of the cross arm, $R$ is the cross arm radius, $\theta$ is the angle between the swinging rod and the vertical direction, $\varphi$ is the rotation angle of the cross arm, $g$ is the gravity, $J_{C}$ is the moment of inertia of the swinging part to the end point of the cross arm. In this system, $\theta$ is the indirect control parameter and $\ddot{\varphi}$ is the direct control parameter.

The simplified model is only used for controller design. When simulating, the Simulink plugin exported by Adams is taken as the inverted pendulum model which is precise though there is random disturbance. About the model for simulation, it will be covered in more detail in the Simulation section.

\section{Controller Design}

\subsection{Sliding Mode Controller}

The block diagram of SMC control system is show in Figure 3. The control target is to limit the error between $\theta$ and $\theta_{d}$ to a certain range, where $\theta$ is the output angle and $\theta_{d}$ is the target angle.

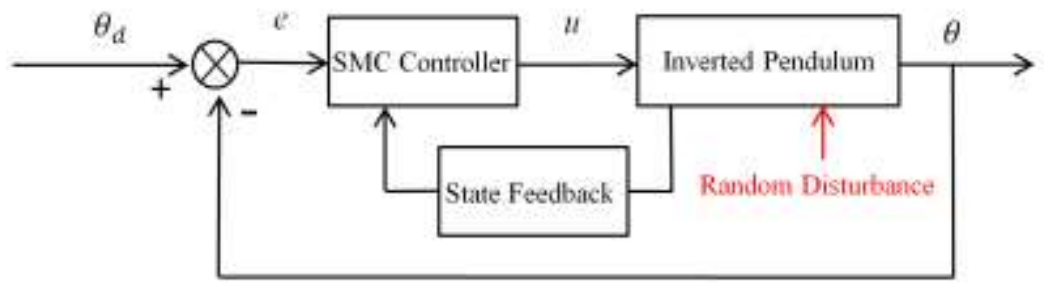

Fig. 3: Block Diagram of SMC Control System.

The Eq. (1) can be standardized for defining dynamic sliding surface which is show in Eq. (2)-Eq. (9).

$$
z_{1}=\varphi+\frac{J_{C}}{m \cdot R_{C} \cdot R} \theta
$$




$$
\begin{gathered}
z_{2}=\dot{\varphi}+\frac{J_{C}}{m \cdot R_{C} \cdot R} \dot{\theta} \\
z_{3}=\theta \\
z_{4}=\dot{\theta}
\end{gathered}
$$

Error equation is show in Eq. (6)-Eq. (9) by bring Eq. (2)-Eq. (5) to Eq. (1).

$$
\begin{gathered}
e_{1}=z_{1} \\
e_{2}=\dot{e_{1}}=z_{2} \\
e_{3}=\ddot{e_{1}}=\ddot{\varphi}+\frac{J_{C}}{m \cdot R_{C} \cdot R} \ddot{\theta}=\frac{\mathrm{g}}{R} \theta \\
e_{4}=\ddot{e_{1}}=\frac{\mathrm{g}}{R} \dot{\theta}
\end{gathered}
$$

Dynamic sliding surface equation is show in Eq. (10).

$$
s=c_{1} \cdot e_{1}+c_{2} \cdot e_{2}+c_{3} \cdot e_{3}+e_{4}
$$

In this equation: $c_{1}>0, c_{2}>0, c_{3}>0$ and the values satisfy the Hurwitze condition.

Bring Eq. (6)-Eq. (9) to Eq. (10) and derivate it then get Eq. (11).

$$
\dot{s}=c_{1} \cdot\left(\dot{\varphi}+\frac{J_{C}}{m \cdot R_{C} \cdot R} \dot{\theta}\right)+c_{2} \frac{\mathrm{g}}{R} \theta+c_{3} \frac{\mathrm{g}}{R} \dot{\theta}+\frac{\mathrm{g}}{R} \cdot\left(\frac{\mathrm{m} \cdot \mathrm{g} \cdot R_{C}}{J_{C}} \cdot \theta-\frac{m \cdot R_{C} \cdot R}{J_{C}} \ddot{\varphi}\right)
$$

According to Eq. (11) the input value ( $\ddot{\varphi}$ ) can be set as shown in Eq. (12).

$$
u=\ddot{\varphi}=\frac{J_{C}}{m \cdot R_{C} \cdot \mathrm{g}}\left(c_{1} \cdot\left(\dot{\varphi}+\frac{J_{C}}{m \cdot R_{C} \cdot R} \dot{\theta}\right)+c_{2} \frac{\mathrm{g}}{R} \theta+c_{3} \frac{\mathrm{g}}{R} \dot{\theta}+\frac{\mathrm{m} \cdot \mathrm{g}^{2} \cdot R_{C}}{R \cdot J_{C}} \theta+M \cdot \operatorname{sgn}(s)\right)
$$

Bring Eq. (12) to Eq. (11) then get Eq. (13).

$$
\dot{s}=-M \cdot \operatorname{sgn}(s)
$$

Take the Lyapunov function as Eq. (14).

$$
V=\frac{1}{2} s^{2}
$$

Derivate the Lyapunov function and bring Eq. (13) to it then get Eq. (15).

$$
\dot{V}=s \cdot \dot{s}<0
$$

The Eq. (15) shows that the Lyapunov function is stable so the system will tends to stabilize under Sliding Model Control [18]. The mechanical structure parameters of the system are show in Table 1. 
Table 1: mechanical structure parameters of the system.

\begin{tabular}{|c|c|}
\hline Mechanical structure parameters & value \\
\hline Equivalent mass of the swinging part $\boldsymbol{m}$ & $0.539(\mathrm{~kg})$ \\
\hline Moment of inertia $\boldsymbol{J}_{\boldsymbol{C}}$ & $0.016043\left(\mathrm{~kg} \cdot \mathrm{m}^{3}\right)$ \\
\hline Cross arm radius $\boldsymbol{R}$ & $0.299(\mathrm{~m})$ \\
\hline Equivalent swinging distance $\boldsymbol{R}_{\boldsymbol{C}}$ & $0.1659(\mathrm{~m})$ \\
\hline Control gain $\boldsymbol{M}$ & 60 \\
\hline Gravity $\mathbf{g}$ & $9.8\left(\mathrm{~m}^{2} / \mathrm{s}\right)$ \\
\hline Sliding surface parameters $\boldsymbol{c}_{\mathbf{1}}, \boldsymbol{c}_{\mathbf{2}}, \boldsymbol{c}_{\mathbf{3}}$ & $27.0,27.0,9.0$ \\
\hline
\end{tabular}

\subsection{Fuzzy Sliding Mode Controller}

The block diagram of Fuzzy SMC control system is show in Figure 4. A Fuzzy Inference Controller is designed to adjust the thickness of the boundary layer in real time which can significantly reduce the chattering near the equilibrium position.

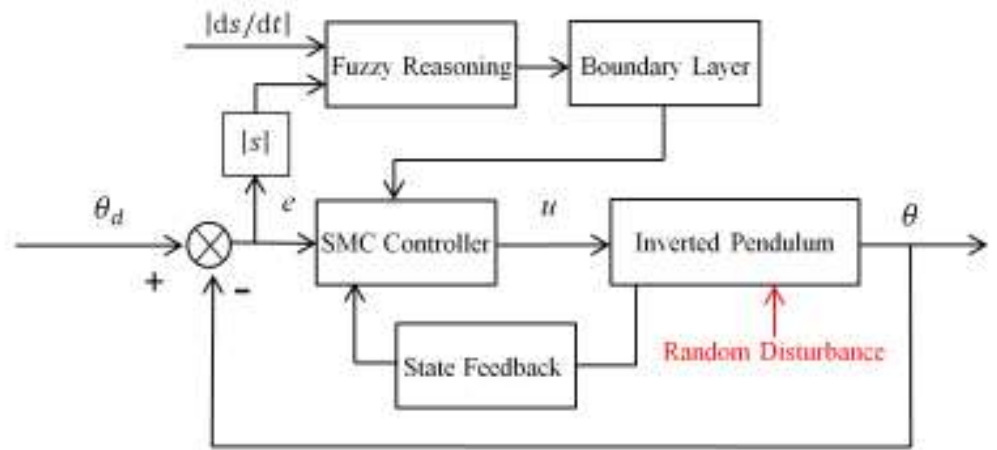

Fig. 4: Block Diagram of SMC Control System.

The definition of boundary layer thickness is shown in Eq. (16).

$$
\Delta=n \cdot \Delta_{1}+(1-n) \Delta_{2}
$$

Where $\Delta_{1}, \Delta_{2}$ are the limit range areas of the boundary layer thickness and the parameters $\left(n, \Delta_{1}, \Delta_{2}\right)$ satisfy the inequalities: $0 \leq n \leq 1,0<\Delta_{1}<\Delta_{2}$. Fuzzy Reasoning Controller adjusts the thickness of the boundary layer by changing the value of $n$.

Three subsets are defined for fuzzy controller input: small (S), middle (M) and big (B). Five subsets are defined for output: big negative (NB), small negative (NS), zero (ZR), small positive (PS) and big positive (BS). The membership functions of input and output are shown in Figure 5. 


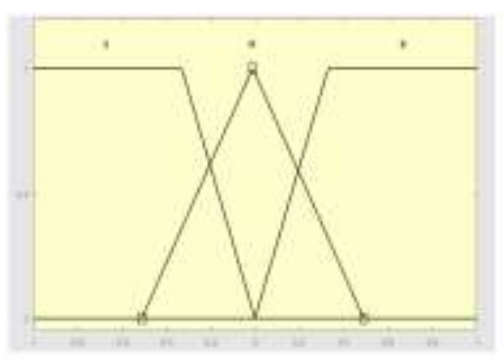

(a) Membership of $s$

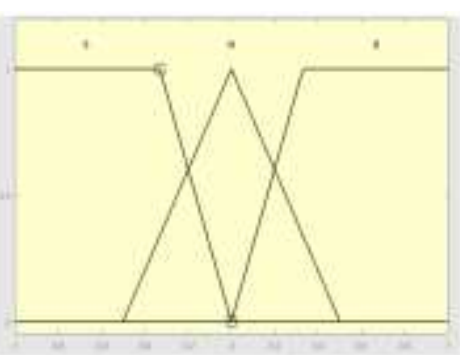

(b) Membership of $\dot{s}$

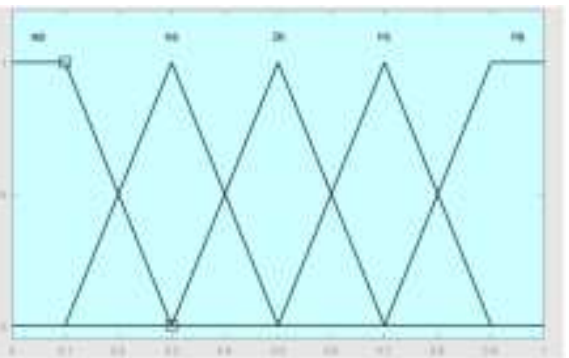

(c) Membership of $n$

Fig. 5: Membership functions of input and output.

The fuzzy inference system and fuzzy surface are shown in Figure 6.

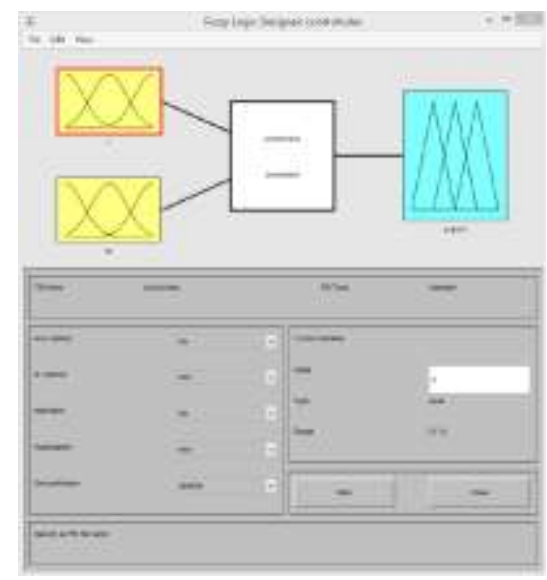

(a) Fuzzy Inference System

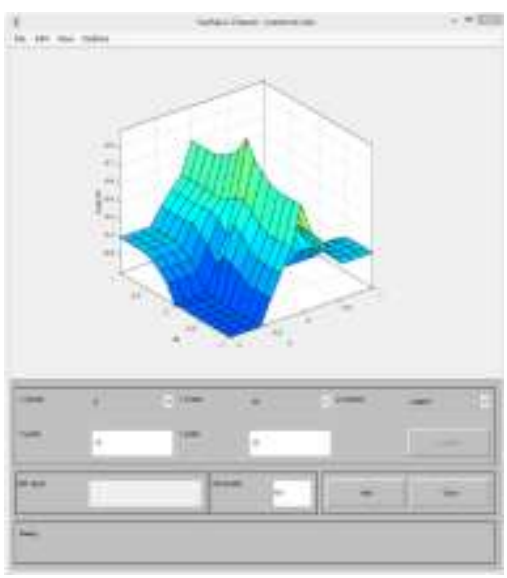

(b) Fuzzy Surface

Fig. 6: Fuzzy Inherence System and Fuzzy Surface.

The principles of adjusting the thickness of the boundary layer can be summarized as:

(1) When the value of $s$ is big, the thickness of the boundary layer should be reduced to increase the control gain which can accelerate the movement of the system state trajectory to the sliding surface and increase the control efficiency.

(2) When the value of $s$ and $\dot{s}$ are small, the thickness of the boundary layer should be increased as much as possible to reduce the chattering caused by the big value of gain parameter.

Based on the principles for boundary layer thickness adjustment, the fuzzy rules are designed and shown in Table 2.

Table 2: Fuzzy Tuning Rules of $n$.

\begin{tabular}{|c|c|c|c|}
\hline $\boldsymbol{n}$ & $\mathbf{S}$ & $\mathbf{s}$ & $\mathbf{B}$ \\
\hline $\boldsymbol{s}$ & NB & NB & NS \\
\hline $\mathbf{M}$ & PS & PB & PS \\
\hline B & NS & NB & NB \\
\hline
\end{tabular}

\section{Simulation Results and Analysis}

\subsection{Simulation Environment}

The simulation environment is built by combining software MATLAB/Simulation and Adams. Adams is an application software for virtual mechanical system analysis which can be used for static, kinematic and dynamic simulation. Meanwhile, it is a good secondary development tool platform due to its open structure and multiple interfaces, which combines the 
advantages from different software such as NX and MATLAB/Simulink. Adams receives the imported mechanical structure from NX10.0 and then the exports MATLAB/Simulink plugin after adding the input and output parameters to the structure. The Adams mechanical structure and the MATLAB/Simulink plugin are shown in Figure 7.

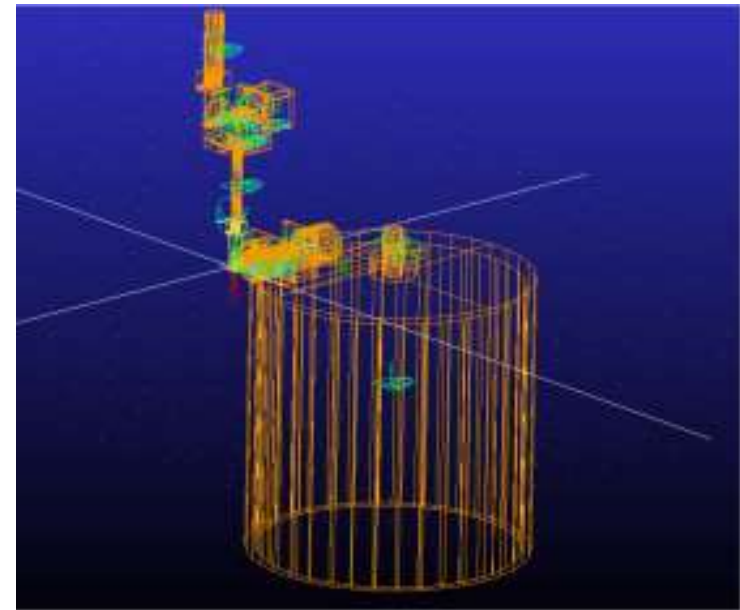

(a) Mechanical Structure in Adams

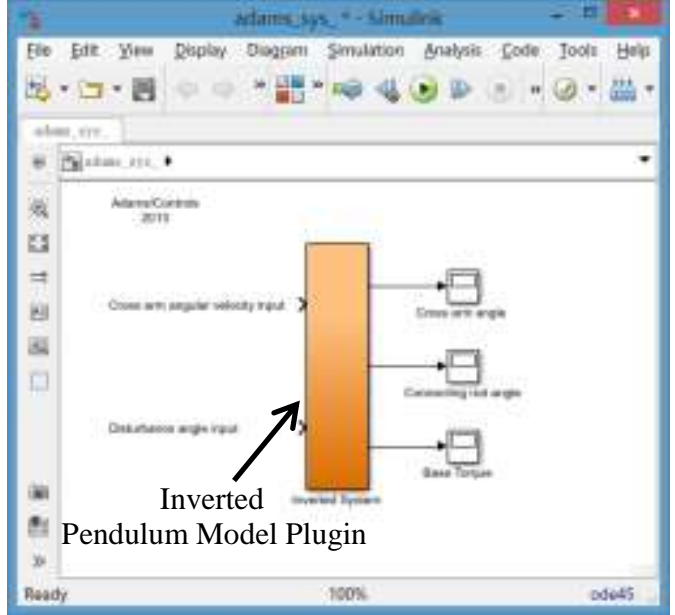

(b) MATLAB/Simulink Plugin

Fig. 7: Adams Mechanical Structure and MATLAB/Simulink Plugin.

When the simulation is in progress, Adams will receive the inputs from the MATLAB/Simulink and returns the outputs back. At the same time, the real-time movements will display in Adams.

The mechanical structure model in Adams will change according to the current status which can guarantee the accuracy of the model and the reliability of the simulation results.

\subsection{PID Controller Simulation}

The simulation results about PID Controller are shown in Figure 8. And the disturbance is added after 5s by rotating the disturbance rod at the constant speed of $1.2 \mathrm{rad} / \mathrm{s}$ and stopping at the angel of $97^{\circ}$, of which the curve is embed in Figure 8 . Besides, the disturbance in SMC Simulation and Fuzzy SMC Simulation is the same as in PID Simulation.

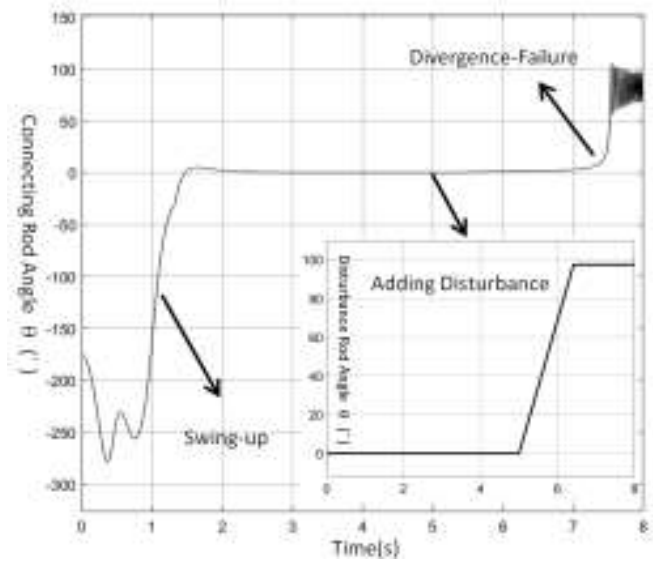

(a) Angle of Connecting Rod

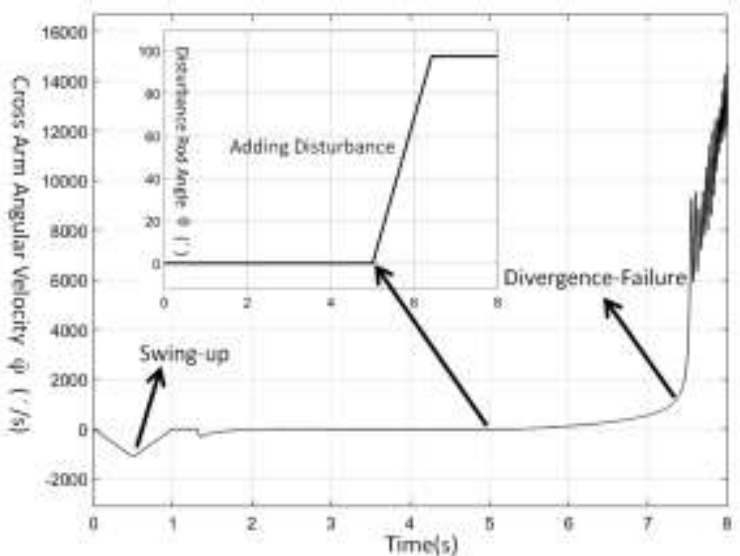

(b) Angular Velocity of Cross Arm

Fig. 8: PID-Controller Simulation.

The parameters of PID Controller: $k_{p}, k_{i}, k_{d}$ are respectively set to $10.0,100.0$ and 2.0. The angel of connecting rod and the angular velocity of cross arm start diverging at $7 \mathrm{~s}$, which proves that PID Controller is unable to achieve the control effect when the model is under random disturbance. 


\subsection{Sliding Mode Controller (SMC) Simulation}

The simulation results about SMC are shown in Figure 9.

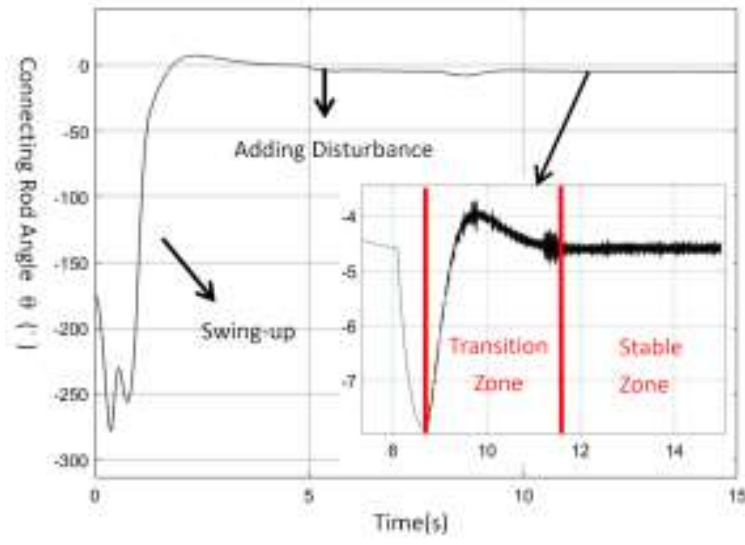

(a) Angle of Connecting Rod

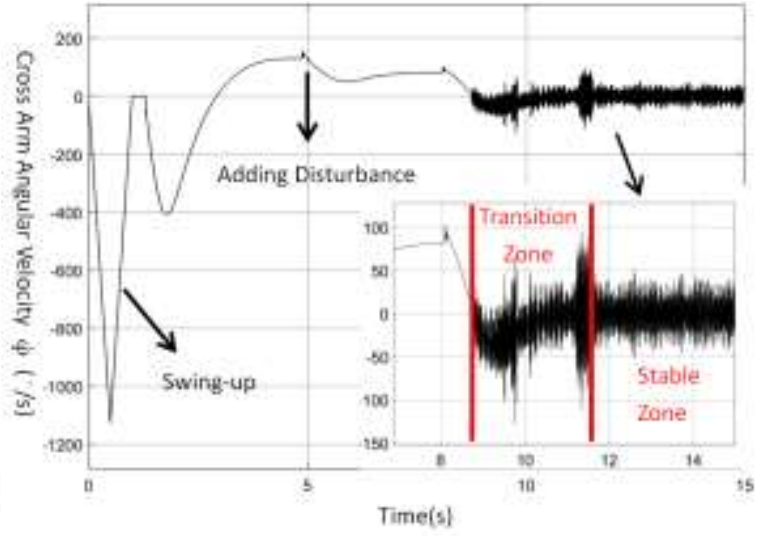

(b) Angular Velocity of Cross Arm

Fig. 9: SMC-Controller Simulation.

The disturbance added to SMC is the same as to PID. The parameters of SMC: $c_{1}, c_{2}, c_{3}, \Delta_{1}, \Delta_{2}$ are respectively set to 27.0, 27.0, 9.0, 0.1, 5. As shown in Figure 9(a), the angle of connecting rod is nearly stable at $-4.6^{\circ}$ with chattering of which the amplitude is about $0.4^{\circ}$. From Figure $9(\mathrm{~b})$, the angular velocity of cross arm is nearly stable at $0 \% \mathrm{~s}$ with chattering of which the amplitude is about $80 \%$ s. Besides, the transition starts at $8.8 \mathrm{~s}$ and stabilizes at $11.5 \mathrm{~s}$.

In summary, the SMC can prevent the inverted pendulum from falling when there is random disturbance, which shows the better robust than PID Controller. But the strong chattering near the equilibrium position may cause damage to the mechanical system.

\subsection{Fuzzy Sliding Mode Controller Simulation}

The simulation results about Fuzzy SMC are shown in Figure 10.

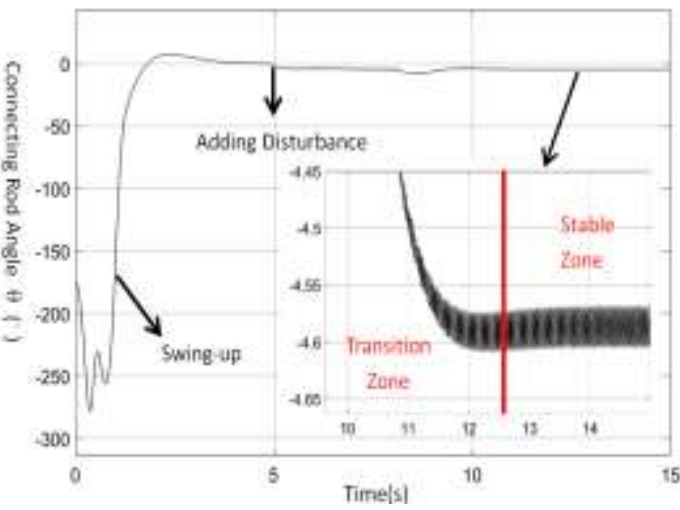

(a) Angle of Connecting Rod

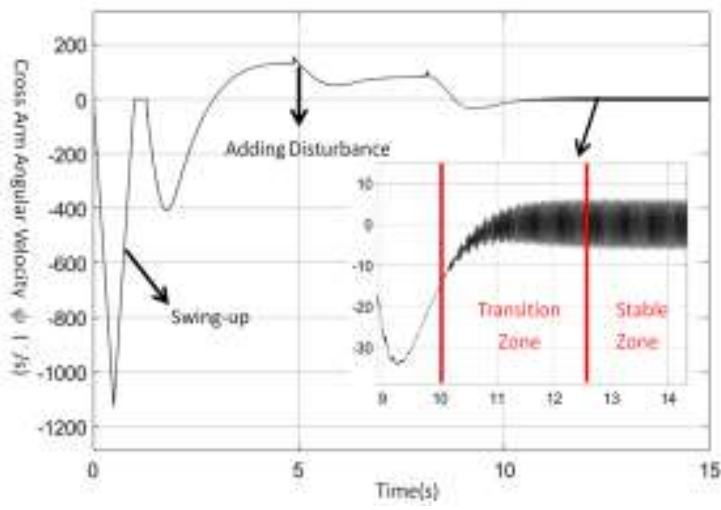

(b) Angular Velocity of Cross Arm

Fig. 10: Fuzzy SMC-Controller Simulation

The parameters and disturbance added to Fuzzy SMC are the same to SMC. As shown in Figure 10(a), the angle of connecting rod is nearly stable at $-4.58^{\circ}$ with chattering of which the amplitude is about $0.04^{\circ}$. From Figure $10(\mathrm{~b})$, the angular velocity of cross arm is nearly stable at $0 \% \mathrm{~s}$ with chattering of which the amplitude is about $10 \%$ s. Besides, the transition zone starts at $10 \mathrm{~s}$ and stabilizes at $12.5 \mathrm{~s}$.

Comparing with SMC, Fuzzy SMC controller can effectively reduce chattering. For the angle of connecting rod $\theta$ reduces from $0.4^{\circ}$ to $0.04^{\circ}$, probably one tenth of the original. And the angular velocity of cross arm $\dot{\varphi}$ reduces from $80 \% \mathrm{~s}$ to $10 \% \mathrm{~s}$, about one eighth of the original. However it takes more time about 1s for Fuzzy SMC to let the system reach the dynamic 
stability. The response speed of the system has decreased, which caused by the real-time adjustment of control parameter gain to weak the chattering.

\section{Conclusion}

In this paper, a new inverted pendulum model with a motor-driven random disturbance rod is proposed. A simplified mathematical model is built for controllers design. Three controllers: PID Controller, Sliding Mode Controller and Fuzzy Sliding Model Controller are adopted to control the inverted pendulum under random disturbance. By combing the software MATLAB/Simulink and Adams, the control results are well displayed. From the control result, the PID Controller is unable to achieve the control objectives and the SMC Controller can meet the control requirement however with strong harmful chattering near the equilibrium. By dynamically changing the thickness of the boundary layer through Fuzzy Control, the Fuzzy SMC Controller can effectively reduce the chattering on the basis of SMC. As for the control rapid decline caused by the boundary layer thickness change in Fuzzy SMC, it can be adjusted by changing the fuzzy rules or related fuzzy parameters to find the balance between reducing chattering and increasing control speed.

\section{References}

[1] Y. W. Wang, K. X. Xing, J. Ma, W. A. Zhang, "Self-disturbance control method and implementation of linear inverted pendulum," J. Control Engineering, vol. 24, no. 04, pp. 711-715, 2017. (In Chinese)

[2] Shuuji Kajita, Mitsuharu Morisawa, Kanako Miura, Shin'ichiro Nakaoka, Kensuke Harada, Kenji Kaneko, Fumio Kanehiro, Kazuhito Yokoi, "Biped walking stabilization based on linear inverted pendulum tracking," in the 2010 IEEE/RSJ International Conference on Intelligent Robots and Systems, Taipei, Taiwan, 2010.

[3] Hyun Wook Kim and Seul Jung, "Experimental studies of controller design for a car-like balancing robot with a variable mass," Korean Institute of Intelligent Systems, vol. 20, no. 04, pp. 469-475, 2010.

[4] Reza Olfati-Saber, "Normal forms for underactuated mechanical systems with symmetry," IEEE Transactions on Automatic Control, vol. 47, no. 02, pp. 304-308, 2002.

[5] Z. Wei, H. Rock, "Energy and passivity based control of the double inverted pendulum on a cart," in Proceedings of the 2001 IEEE International Conference on Control Applications, Mexico City, Mexico, 2001.

[6] Tobias Glück, Andreas Eder, Andreas Kugi, "Swing-up control of a triple pendulum on a cart with experimental validation," Automatica, vol. 49, no. 03, pp. 801-808, 2013.

[7] Wang J J, "Simulation studies of inverted pendulum based on PID controllers," Simulation Modelling Practice and Theory, vol. 19, no. 01, pp. 440-449, 2011.

[8] Wei Q F, W. P. Dayawansa, W. S. Levine, "Nonlinear controller for an inverted pendulum having restricted travel," Automatic, vol. 31, no. 06, pp. 841-850, 1995.

[9] Wang L X, "Stable adaptive fuzzy controllers with application to inverted pendulum tracking," IEEE Transactions on Systems, Man, and Cybernetics, Part B: Cybernetics, vol. 26, no. 05, pp. 667-691, 1996.

[10] G. A. Medrano-Cersa, "Robust computer of an inverted pendulum," IEEE Control Systems, vol. 19, no. 03, pp. 58-67, 1999.

[11] C. W. Anderson, "Learning to control an inverted pendulum using neural networks," IEEE Control Systems Magazine, vol. 09, no.03, pp. 31-37, 1989.

[12] Yang P, Ma J, Sun W, Liang L H, Li G B, "Simulation and research of the fuzzy sliding mode controller of the ship test bed," in Proceedings of the 2006 International Conference on Machine Learning and Cybernetics, Dalian, China, pp 457-462, 2006.

[13] Otto Cerman, Petr Hušek, "Adaptive fuzzy sliding mode control for electro-hydraulic servo mechanism," Expert System with Applications, vol. 39, no. 11, pp.10269-10277, 2012.

[14] Han Y Z, Xiao H R, Pan W G, Wang C S, "A fuzzy sliding mode controller and its application on ship course control," in 2010 Seventh International Conference on Fuzzy Systems and Knowledge Discovery, Yantai, China, 2010.

[15] Yuan X H, Chen Z H, Yuan Y B, Huang Y H, "Design of fuzzy sliding mode controller for hydraulic turbine regulating system via input state feedback linearization method," Energy, vol. 93, no. 1, pp. 173-187, 2015.

[16] Hossein Rouhani , Caro Lucas , Rasoul Mohammadi Milasi , Mansour Nikkhah bahrami, "Fuzzy sliding mode control applied to low noise switched reluctance motor control," in 2005 International Conference on Control and Automation, Budapest, Hungary, 2005. 
[17] Triet Hung Ho, Kyoung Kwan Ahn, "speed control of a hydraulic pressure coupling drive using an adaptive fuzzy sliding mode control," IEEE/ASME Transactions on Mechatronics, vol. 17, no. 05, pp. 976-986, 2012.

[18] Liu J K, Basic theory and design method of sliding mode variable structure control MATLAB simulation, Beijing: Tsinghua University Press, 2015. 